\title{
Eye SCRUTinized WHEel Chair For PEOPLE AFFECTED WiTH TETRAPLEgIA
}

\author{
Mrs.B.Buvanswari ${ }^{1}$ and Dr. T.Kalpalatha Reddy ${ }^{2}$ \\ ${ }^{1}$ Research Scholar, Department of Computer Science Engineering, Sathyabama \\ University, Chennai. \\ ${ }^{2}$ Dean R \& D, Department of ECE, S.K.R Engineering College, Chennai
}

\begin{abstract}
Nowadays the requirement for developing a wheel chair control which is useful for the physically disabled person with Tetraplegia. This system involves the control of the wheel chair with the eye moment of the affected person. Statistics suggest that there are 230,000 cases of Tetraplegia in India. Our system here is to develop a wheelchair which make the lives of these people easier and instigate confidence to live in them. We know that a person who is affected by Tetraplegia can move their eyes alone to a certain extent which paves the idea for the development of our system. Here we have proposed the method for a device where a patient placed on the wheel chair looking in a straight line at the camera which is permanently fixed in the optics, is capable to move in a track by gazing in that way. When we change the direction, the camera signals are given using the mat lab script to the microcontroller. Depends on the path of the eye, the microcontroller controls the wheel chair in all direction and stops the movement. If there is any obstacle to be found before the wheel chair the sensor mind that and it stop and move in right direction immediately. The benefit of this system is too easily travel anywhere in any direction which is handled by physically disabled person with Tetraplegia.
\end{abstract}

\section{KEYWORDS:}

Daugman's, Eye movement, Electronic wheel chair, Microcontroller, Tetraplegia.

\section{INTRODUCTION}

The spinal cord injury occurs which means the failure of sensation or movement to some extent in the legs, bowel, bladder, and sexual region is referred as Tetraparesis .Paraplegia is similar but does not affect the arms. The loss is usually sensory and motor, which means that both sensation and control are lost. Tetraparesis, on the one-time hand, which means muscle failing affecting all four limbs. It may well be flaccid or spastic. Tetraplegia is root cause by damage to the brain or the spinal cord at a high level in particular, spinal cord injuries secondary to the cervical spine . The injury, which is identified as a lesion, causes sufferers to lose biased or total function of all four limbs, significance the arms and the legs. Tetraplegia is defined in many ways, usually affects arm movement supplementary so than a other can injury. On the other hand, all tetraplegics have some kind of finger dysfunction. For person with this disability, many different kind of electrical and robotic wheelchairs have been designed. These people have troubles to use a predictable wheelchair. A current clinical review indicated that $9 \%-10 \%$ of patients who received power wheelchair training establish it tremendously complicated or impossible to use it for their actions of daily livelihood, and $40 \%$ of patients found the direction-finding tasks difficult

DOI : $10.5121 /$ ijcseit.2015.5202 
or impossible. These people are needy others to move forward them, so that they often feel powerless and out of control. An eye tracking method is proposed here. The design of eye monitored wheel chair was stimulated by past system such as hand over gesture based wheel chair which involves the direction-finding of wheel chair using hand over gestures. In that system the hand movements of the disabled person was used to guide the wheel in the desired direction. We required to proposing our system by devious wheel chair for people affected with Tetraplegia and provided that more independency. A camera is provided that to user for controlled by their eye movements. By the eye movements the wheel chair will drive in all direction with accurate way.

\section{Problem Statement}

The vision-controlled wheelchair is not easy to organize. The present system to control eye movement and the suitability of position correction will have an effect on the systematic control. However, investigate on maimed supplementary instrument still has positive significance in provided that the handicapped chance to acquire greater mobility. So we proposed an eye movement controlled based wheel chair for physically challenged persons to travel in any desirable direction.

\section{LITERATURE SURVEY}

The employment of these(i.e. used difficult input devices) has caused inconvenience to the physically disabled person. In this system, a computer input device by human eyes only is proposed for handicapped person and also for wearable computing. There are various types of wheel chair in the existing system.

\section{Hand Gesture Based Wheelchair Movement:}

A wheel chair control which is used for the physically disabled person with his hand movement or his hand gesture recognition by using Acceleration technology. It has two operation modes which are based on head movements: Mode 1 uses simply one head movement to give the command to control, and Mode 2 employs one to four head movement. An EEG tool, namely Emotiv EPOC, have been deployed in this HMI to attain the head movement information of users.

\section{Drawbacks:}

It requires human effort to navigate devices which proves to be difficult for people with deformities.

\section{Voice Operated Wheel Chair}

The wheel chair is controlled with the voice command from the handicapped person. Voice command is given from android mobile and transformed into the text and this text is given to microcontroller via bluetooth component to control the operation of DC motors. A handicapped person still with Legs and Hand can use this and become autonomous. Some of the advantages identified are rapidly changing input can be easily recognized due to android mobile, suitable for 
practical application as compared to HM 2007,requires less wiring because of Bluetooth section, less hardware requirement i.e. compact, economical, reduces manpower and user friendly .

\section{Drawbacks:}

- Less accurate on

- Background Noise

- Speaker variability

- Speaking style

- Speed of the speech.

\section{Implementation of Head and Finger Based Automated Wheel Chair:}

A head and finger based controlled assist system is developed to control the motor rotation of wheel chair through head and finger movement of physically challenged person. On basis of the head and finger movements the accelerometer and the flex sensor will force the motor fitted to the wheel chair. It will be able to driven in any of the four directions. The automatic wheelchair works on simple electronic control system and the automatic arrangement that is controlled by a Programmable Interface Controller.

\section{Drawbacks:}

$\checkmark \quad$ It proves to be a burden to user.

$\checkmark \quad$ The flex sensor value was predictable to be within a small value, only few combinations have been brought.

$\checkmark$ Hardware has also been done to validate this technology.

\section{Eye Movement Tracking For Wheelchair Control:}

This system proposes a new algorithm called coherence algorithm, for eye motion tracking. This system presents an function of eye tracking process to such wheelchairs. The coherence algorithm tracks the movement of eye in moreover left or right direction. The eye movement discontinuous feature is also used by the algorithm to organize the starting and stopping of wheelchair. This is used as the inspiring factor for the improvement of our system.

\section{Proposed Methodology}

\section{System Overview}

Consists of three main parts:

$\checkmark$ A camera connected to the laptop system that will track the camera wearer's eye.

$\checkmark$ The microprocessor will receive a USB output from the laptop and translate the digital output to electric signals that will be sent to the wheelchair for movement through the microcontroller chip.

$\checkmark$ A signal activate Wheel Chair 


\section{General Architecture}

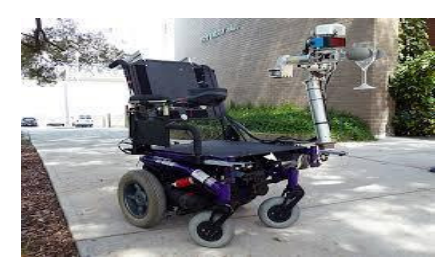

Figure.1 General Architecture for proposed System

In Image Capturing component, we capture the image and after perfect capturing of image it send to Image investigation Module where segmentation of image is done and dealing out of finding the pupil and its path is found out. In the image analysis model, we separate the iris and the segmentation of the patient's eye using Hough Transform.

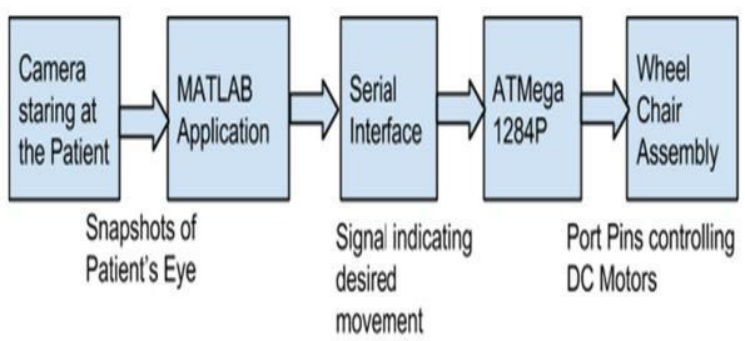

Figure.2. Overall System Process

\section{Camera Staring At The Patient :}

It involves two functions namely,

- Image Capturing Method

- Image Processing Method

\section{Image Capturing System}

Image Capturing is to capture a series of iris images from the topic using a specially designed camera. In iris identification image capturing is a very important step. In sight of the fact that iris is small in size and dark in color, it is not easy to acquire good image. The image is then altered from RGB to gray level for more processing. It is to capture a series of iris images from the topic using a specifically arranged camera. A standard diameter of $12 \mathrm{~mm}$, a camera be required to have sufficient motion to capture the details of the iris pattern.

\section{Image Processing System}

The role of segmentation is to remove non useful information, exclusively the pupil part and the part outside the iris (sclera, eyelids, skin). Daugman recommended an integrodifferential operator to position both the pupil and the iris outline. The algorithm will carry out the iris recognition in two phases. In the first phase, uses the facts that a pupil is a very dark blob of certain minimum 
size picture, and no additional segment of continuous dark pixels are of the same size. The algorithm discovers the center of the pupil and the two radial coefficients as the pupil is constantly a perfect circle. The second algorithms receive the information of the pupil canter and try to find direction in which the eye looks.

- Discover the center of the pupil .

- Discovery of the track in which eye looks.

\section{Discovery of the track in which eye looks}

The arrangement of the process that was diminish the complexity obviously by scale down every part of images to a constant image size to speed up the entire process. First step is to compute the Gaussian blur function. Gaussian blur is the outcome of blurring an image by a Gaussian function.

Mathematically, concern a Gaussian blur to an image is the similar as convolving the image with a Gaussian function. This is always called as a two-dimensional Weierstrass transform. The Gaussian blur is a category of image-blurring filters uses a it function for compute the conversion to be significant to each pixel in the image.

The Gaussian function's equation in one dimension is Where $\mathrm{x}$ is the distance from the origin in the horizontal axis, origin distance in vertical axis is $y$, and the standard deviation of the Gaussian distribution is .

$$
G x=12 \pi \sigma 2 e-x 22 \sigma 2(1)
$$

Applied in two dimensions, this method produces a surface whose contours are concentric circles from the centre point. The principles from this distribution are used to build a convolution matrix and it is applied to the original image. The value of each pixel is set to new weighted average of that pixel's neighborhood.

The original pixel's value receives the heaviest weight from the Gaussian and neighboring pixels get smaller weights as their distance to the original pixel raise. This results in a blur that preserve boundaries and edges improved than other, more consistent blurring filters. Pupil location is found by algorithm of daugman .Using this we will support the location of pupil and get the coordinate of the centre of the pupil. Method suggested by daugman is

$$
(r, 0, y 0) G \sigma \bar{r} * \partial \partial r(x,) 2 \pi r r, x 0, y 0 d s(2)
$$

Where G(r) function represents Gaussian Filter the image is scaled to sigma size. It is the balancing level function blurred at level set by sigma, penetrating iteratively for maximal contour vital derived at consecutively greater scales of analysis throughout the three parameter space of centre coordinates and radius $\mathrm{x} 0, \mathrm{y} 0$ and $\mathrm{r}$ values. The equation thus becomes as

$$
\max r, 0, y 0=\partial \partial r I(r * \cos \theta+x 0, r * \sin \theta+y 0) 2 \pi 0(3)
$$

The specialty of this equation is even when the eye is at certain phase angling it can identify the 
center of the pupil, this is because line integration is inspection the maximum gradient circle in that particular center values. It is a particular spot it going to verify at what angle the gradient is highest. After achieved the center coordinates of both iris and pupil we make sure both circles doesn't make bigger and stays surrounded by the other is inside iris circle and pupil circle should be there and therefore determined that the obtained circles are accurate and if fails then resume of the entire process takes place.

\section{Identifying the direction of the pupil}

The image is given to the MATLAB tool , then it will processing and generate an output image with its coordinates of iris and pupil. The output image size will be $320 * 240$. The image output will arrange in the center block and it will indicate the pupil position as straight. While the block at second row and third column should specify the position of pupil as left

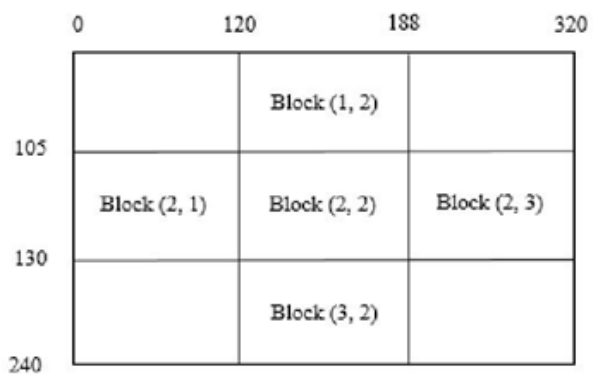

Figure.3. Sample process of image capturing module

and in the same way the block at second row and first column should indicate the pupil location as right and the block at first row and second column should specify the location of pupil as top and the block at third row and second column should point out the location of pupil as bottom.

The output image getting from mat lab software after processing it will be subdivided into nine blocks by fixing the coordinates of each block. The output images with standard size already specified will be considered as a rectangle with length of 320 and its breadth is 240 . Since the rectangle breadth is accountable for dividing it into three rows, 240 should be divided into three parts. But in order to diminish errors, thee center or second row is specified in a smaller width. Likewise the length of the rectangle is accountable for dividing it further into three columns with three parts. To decrease errors the center or second part is 


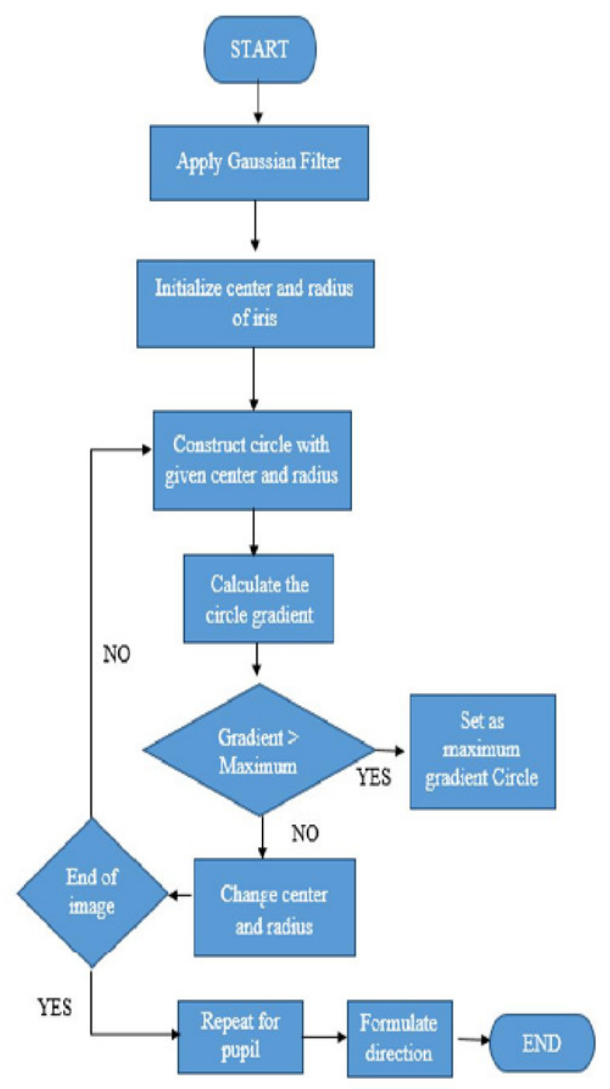

Figure.4 Proposed Direction Detection Technique

prepared of smaller width. The coordinates of every output block is shown below.

Block $(1,2)$ : The iris output coordinates are satisfying below constraints will discover the pupil position as top.

Condition: if $((\mathrm{x}>120$ and $\mathrm{x}<188) \& \& \mathrm{y}<105))$

Block $(2,1)$ : The iris output coordinates are satisfying below constraints will discover the pupil position as Right.

Condition: if $((\mathrm{y}>105$ and $\mathrm{y}<130) \& \& \mathrm{x}<120))$ Block $(2,2)$ : The iris output coordinates are satisfying below constraints will discover the pupil position as Straight.

Condition: if $((\mathrm{x}>120$ and $\mathrm{x}<188) \& \&(\mathrm{y}>105$ and $\mathrm{y}<130))$

Block $(2,3)$ : The iris output coordinates are satisfying below constraints will discover the pupil position as Left.

Condition: if $(x>188 \& \&(y>105$ and $y<130))$ Block $(3,2)$ : The iris output coordinates are satisfying below constraints will discover the pupil position as Bottom. 
Condition: if $((\mathrm{x}>120$ and $\mathrm{x}<188) \& \& \mathrm{y}>105))$

\section{Working Principle:}

After getting the input from the source, we apply the Gaussian Process because it is very commonly used filter to reduce noise, which means fully described by its mean and covariance function. GPs are used to infer, or guess, function values at a finite set of guess points from the observed sample data set. The weights are samples of the Gaussian function where $\boldsymbol{\sigma}$ controls the amount of smoothing. As $\boldsymbol{\sigma}$ rises, more model must be obtained to stand for the Gaussian function accurately.

$$
G_{\sigma_{1}}(x, y)=\frac{1}{2 \pi \sigma_{1}^{2}} e^{-\frac{x^{2}+y^{2}}{2 \sigma_{1}^{2}}}
$$
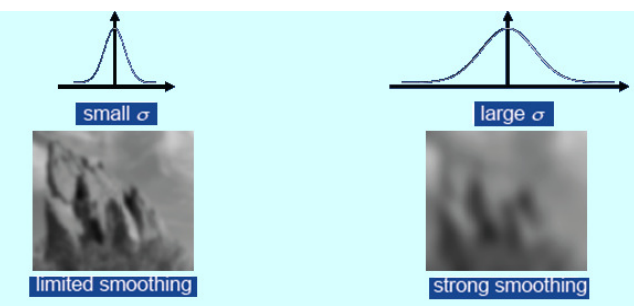

Figure 5. Smoothing filters by Gaussian

For example smoothing filters,

After applying the Gaussian filter, we are initialize the center and radius of iris. Then build the sphere based on the given midpoint and radius of the iris. To find out the circle gradient, if its value is greater than 1 , then set as maximum gradient circle . or else change the center and its radius then repeat the same process discussed above. Finally formulate the direction exactly.

\section{Implementation of Daughman's Algorithm and Results}

1. In fig.6. shown below the input and its processing images are produced after using this algorithm .The location of iris and pupil is detected and it can be highlighted through the pointed circle as shown below. The location of the image is discovered and then the decision for the given image should produced as left

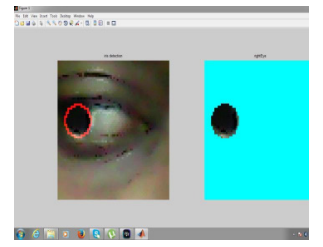

Figure.6. the input of the eye starring in the left and the corresponding output

2. In fig.7. shown below the input and its processing images are produced after using this algorithm .The location of iris and pupil is detected and it can be highlighted through the pointed circle as shown below. The location of the image is discovered and then the decision for the given image should produced as Right. 


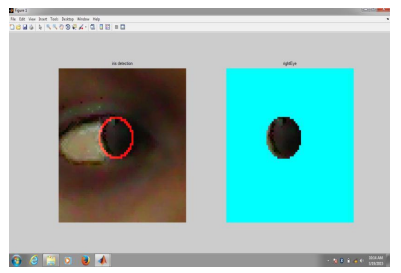

Figure. 7 The input of the eye starring in the right and the corresponding output

3. In fig.8. shown below the input and its processing images are produced after using this algorithm .The location of iris and pupil is detected and it can be highlighted through the pointed circle as shown below. The location of the image is discovered and then the decision for the given image should produced as Straight

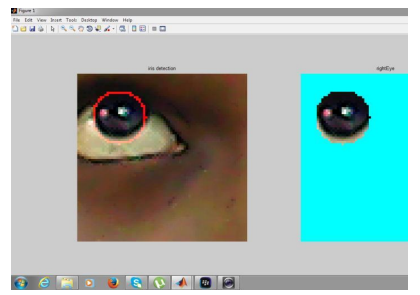

Figure. 8 The input of the eye starring in the Straight and the corresponding output

We are assigned the binary value for basic direction to control wheel chair as follows initially.

100 - Designate the wheelchair movement as in a straight line by bright the First LED named as D1.

010 - Point out the wheelchair movement as Left by bright the Second LED named as D2.

110 - identify the wheelchair movement as Right through glowing third LED named as D3.

\section{CONCLUSION}

We have presented a wheel chair system for the people affected by Tetraplegia. The wheel chair which is decides here employ the Daughman's algorithm and wrapping up of direction in which pupil seem to be decided by fixing range to the particular direction as user looks. Deduction of the pupil works even if the image is taken in little dark environment. The image capture process method, eye movement recognition process and its algorithm for authenticate movement effort execute very consistently as our results suggest. The aim of the presented method is to contribute to the people suffering from Tetraplegia in our smallest way by setting out an idea for a system which could actually better the lives of millions of people across the globe and instill confidence in them. Finally, we have done honesty to the idea, and ended up getting more than believable results. The motive of our project is to provide a source of confidence in the physically challenged person and help them to lead an independent life. 


\section{REFERENCES}

[1] Q.X. Nguyen and S.Jo,"Electric wheelchair control using head pose free eye-gaze tracker", Electronics Letters, Vol. 48 No.13, 21st June 2012

[2] Djoko Purwanto, Ronny Mardiyanto, Kohei Arai, "Electric wheelchair control with gaze direction and eye blinking", Artif Life Robotics, 14:397-400, May 18, 2009

[3] Er. Vijay Dhir \& Dr.(Ms) Maitryee Dutta., (2009),'New Method of IRIS RECOGNITION Based on J.Daugman'sPinciple', Second InternationalConference on Emerging Trends in Engineering and Technology, 09.

[4] Jayesh K. Kokate1, A. M. Agarkar2 1Student ME, 2Professor, Dept. of Electronics \& Telecommunication, SSGMCE "VOICE OPERATED WHEEL CHAIR", Volume: 03 Issue: 02 I Feb2014

[5] Prof. Vishal V. Pande, Nikita S.Ubale, Darshana P. Masurkar, Nikita R. Ingole, Pragati P. Mane, "Hand Gesture Based Wheelchair Movement Control for Disabled Person Using MEMS"., ISSN : 2248-9622, Vol. 4, Issue 4( Version 4), April 2014, pp.152-158

[6] S. Tameemsultana and N. Kali Saranya, Bonfring "Implementation of Head and Finger Movement Based Automatic Wheel Chair" International Journal of Power Systems and Integrated Circuits, Vol. 1, Special Issue, December 2011.

[7] J.S. Han, Z. Zenn Bien, D.J. Kim, H.E. Lee, and J.S. Kim, "Human machine interface for wheelchair control with EMG and its evaluation", In Proc. of the 25th Annual Int. Conf. of the IEEE Engineering in Medicine \& Biology Society, volume 2, pages 1602- 1605. IEEE, 2003.

[8] I. Moon, M. Lee, J. Chu, and M. Mun, "Wearable EMG-based HCI for electric-powered wheelchair users with motor disabilities". In Proceedings of the 2005 IEEE International Conference on Robotics and Automation, pages 2649-2654. IEEE, 2005.

[9] S. Tameemsultana and N. Kali Saranya, "Implementation of Head and Finger Movement Based Automatic Wheel Chair", Bonfring International Journal of Power Systems and Integrated Circuits, Vol. 1, Special Issue, December 2011.

[10] Poonam S. Gajwani1\& Sharda A. Chhabria2, "Eye Motion Tracking for wheelchair Control" International Journal of Information Technology and Knowledge Management July-December 2010, Volume 2, No. 2, pp. 185-187 